\title{
A FRAMEWORK FOR INTERACTIVE GPU-SUPPORTED RENDERING AND STYLING OF VIRTUAL HAIR
}

\author{
Rui (Ray) Zhang and Burkhard C. Wünsche \\ Department of Computer Science, Private Bag 92019, University of Auckland, New Zealand \\ ruizhangnz@yahoo.co.nz,burkhard@cs.auckland.ac.nz
}

Keywords: Hair modelling, interaction techniques, GPU algorithms, wisp model, key strands.

\begin{abstract}
The interactive styling and rendering of virtual hair is essential for creating realistic looking human avatars for use in computer games, virtual worlds, and movie special effects. Hair models can contain tens of thousands of hair strands and hence it is important to develop techniques to modify the hair in a realistic fashion and to render it at interactive frame rates. In this paper we present a GPU-based framework for styling and rendering of virtual hair. We use wisps to represent basic units of hair strands and present an improved statistical model for hair wisp generation which allows the creation of smooth and fringy styles. Fast modelling is achieved by using create, edit, delete and copy and paste operations for key strands representing wisp. The styling process is simplified by using a local coordinate system for hair strands in order to define preferred styling (brushing) directions. Fast photo-realistic rendering is achieved by using the latest GPU functionalities for both the light reflection calculation and the shadow generation of hair strands. We also propose a new method for real-time anti-aliasing using GPU programming.
\end{abstract}

\section{INTRODUCTION}

Computer generated realistic virtual humans are required in applications such as the movie industry (CGI - computer generated imagery), computer games, and as avatars for virtual worlds. An important factor for achieving a realistic human appearance is the development of a realistic hair model. Psychological studies have shown that hair is a determining factor of a person's first impression when meeting his or her counterpart (Lafrance, 2005). Therefore, the styling and rendering of virtual hair is an active field of research in Computer Graphics. Hair styling is challenging since the complex behaviour of each hair strand and the interactions among the hair strands during animation and styling must be controlled in a physically realistic way.

In this paper we present a GPU-based framework for styling and rendering of virtual hair. Section 2 gives an overview of hair modelling techniques and section 3 introduces our wisp based model and an improved statistical model for hair wisp generation. Section 4 presents algorithms for fast photo-realistic rendering using improved data structures and more efficient GPU algorithms for the light reflection calculation and shadow generation. Section 5 proposes methods for real-time antialiasing using GPU programming. Section 6 contains implementation details and section 7 discusses our results. We draw conclusions about our research in section 8 and finish with a listing of future work in section 9 .

\section{INTERACTIVE HAIR MODELLING AND STYLING}

In order to develop efficient styling tools it is important to find the most suitable hair model. Popular approaches are polygonal surfaces, volumetric textures, and strand-based and wispbased models.

Parke introduced a fast and simple way to model hair which uses simple texture mapped polygonal surfaces to capture the shape and appearance of hair (Parke, 1974). Since the surface representation does not model the complex geometry of hair strands the specular lighting effects are not correct and the resulting rendered images lack realism.

In contrast strand-based models represent every hair strand explicitly either by thin cylinders, which are slow to render and suffer from aliasing artefacts, or 
by using connected line segments (Anjyo, Usami, and Kurihara, 1992; Rosenblum, Carlson, and Tripp, 1991). This type of model is suitable for simple styles using long, animated hair, but it is not practical for creating complex hairstyles due to the large number of strands which must be moved.

An improvement is achieved by grouping hair strands into wisps whose shape and movement is defined by using key strands. The idea is based on the observation that adjacent hair strands tend to form wisps due to static attraction and artificial styling products. Daldegan et al. use a triangular head mesh and define key hair strands at each triangle vertex in order to interpolate the wisps' hair strands (Daldegan et al., 1993). Yang et al. use generalized cylinders to represent hair wisps (Yang et al., 2000). Plante et al. propose an animation method to deal with the interactions among wisps and to simulate complex hair motions (Plante et al., 2001). The above three models have the advantage that they make it easy to control hair styling. However, the methods are not effective for controlling complex hairstyles such as curly hair.

In 2002 Kim and Neumann proposed a multiresolution hair modelling system, which can handle fairly complex hairstyles (Kim and Neumann, 2002). The model makes it possible to define the behaviour of hair over the entire range from hair wisps down to individual hair strands. Different hair styles can be created rapidly using high-level editing tools such as curling, scaling and copy/paste operations. Subsequently Choe and Ko introduced a statistical wisp model to generate a wide range of human hairstyles (Choe and Ko, 2005). The authors simulate hair deformation by applying physical properties of hair such as gravity and collisions response. The model is capable of handling a wide range of human hair styles but is unsuitable for simulating hair animation due to a lack of real-time performance of their modelling algorithm and failing in collision detection in some cases.

Other models have been proposed such as volumetric textures for modelling fur and short hair styles (Perlin, 1989; Kajiya and Kay, 1989) and particle-based hair models, which simulate hair strands as trajectories of particles shot from the head (Stam, 1995).

In conclusion wisp-based models are the most flexible hair models since they support high-level operations such as copy/paste between wisps when designing a hair style, but also give control about details of a hair style. Disadvantages of this approach are the large amount of time needed to handle the interactions between hair strands such as collision detection and difficulties in simulating convincing hair animation. However, for many applications with little animation, such as hair styling, the advantages outweigh the disadvantages, and we therefore use a wisp-based model.

\section{STYLING TOOLS}

Our hair model is based on the static wisp-based model proposed by Choe and Ko (2005). Hair strands are placed on a high resolution triangle mesh head model shown in figure 1.

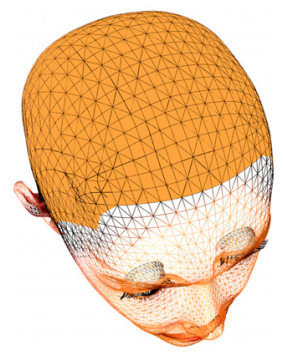

Figure 1: A head model represented by a triangle mesh. The scalp region is coloured brown.

Inspired by Kim and Neumann's interactive hair modelling system (Kim and Neumann, 2002) we added tools to enable users to manipulate wisps. Since a scalp can have tens of thousands of hair strands we need a system to easily control groups of hair strands. The user is able to group several triangles on which to grow a wisp. The size (number of strands) of a wisp is dependent on the area of selected triangles. The smallest wisp is defined by a single triangle. This level of detail is sufficient for defining a wide variety of hair styles since the triangles are small compared to the scalp's area.

The geometry of a wisp is controlled using a key strand as illustrated in figure 2. The key strand defines the geometry for all strands of a wisp which are then obtained by translating them as described in subsection 3.2. We use Catmull-Rom splines (Catmull and Rom, 1974) to represent hair strands since they are smooth $\left(\mathrm{C}^{1}\right.$ continuous) and because they interpolate their control points which makes designing a particular hair style more intuitive. 


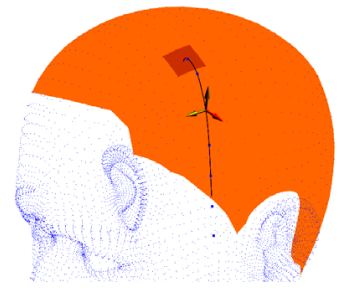

Figure 2: A key strand defining a wisp for a group of four triangles on the scalp. The dark area on the scalp defines the selected triangles. The blue square points are the control points on the key strand.

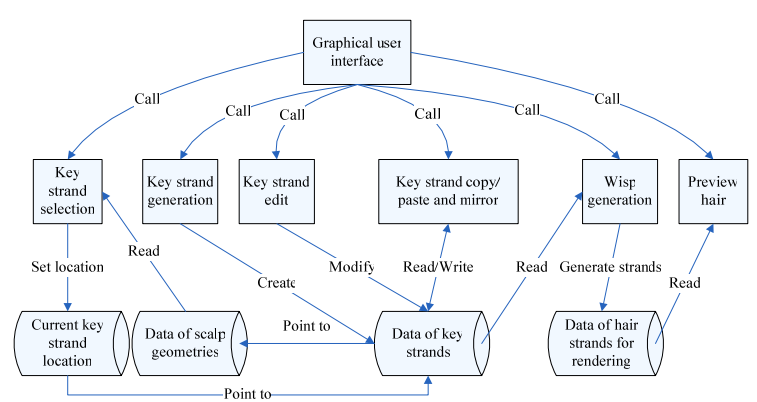

Figure 3: An overview of the components of our hair styling toolset.

The components of our hair styling toolset are illustrated in figure 3 . The key strand selection component enables users to choose triangles to grow a wisp or to select an existing wisp for editing. After a group of triangles has been selected it is recorded together with the location of its current key strand. The location of the key strand is determined as the centre of the first triangle of the selected group of triangles (see section 3). The selection of scalp triangles and strand control points has been implemented with the OpenGL "select" mechanism. This enables us to detect whether the projection of a graphical primitive onto the view plane overlaps with a hit region surrounding the mouse location in which case we select the front most primitive.

\subsection{Key Strand Control}

Users are able to interactively modify a key strand by adding, deleting and moving control points. The interface for changing the $3 \mathrm{D}$ coordinates of the control points of a key strand is illustrated in figure 4. Since the mouse movements on the screen are in $2 \mathrm{D}$ we have to map this into a suitable $3 \mathrm{D}$ motion. A common solution in modelling applications is to restrict movements to the coordinate directions, parallel to the view plane or within a user defined plane.

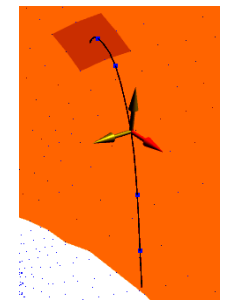

Figure 4: The user interface for changing the 3D positions of a key strand's control points. The red arrow indicates the currently active direction in which the control point can move forward and backward. The yellow arrows indicate the non-active directions.
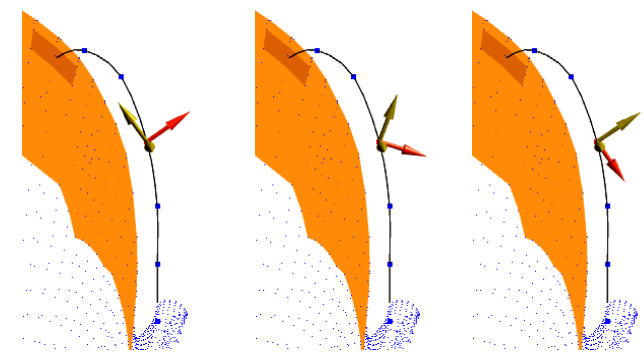

Figure 5: Rotation of the local coordinate system at a control point.

We found that in hair styling the preferred hair movement direction depends on a particular style, e.g. "brushing" hair backwards, lifting it up, pulling it down or curling it. We therefore define for each key strand a local coordinate system of styling directions represented by three orthogonal arrows. The currently active styling direction is indicated by a red arrow. A new styling direction is obtained by choosing one of the non-active arrows or by changing the local coordinate systems as illustrated in figure 5.

Suitable default directions for the local coordinate system at a control point are the curve tangent at that point, the surface normal at the scalp point closest to the control point and the vector perpendicular to these two vectors.

\subsection{Wisp Control}

Users can change the size of a wisp by changing the triangles defining the area on which the strands of this wisp grow. High-level copy/paste and mirror operations between wisps are provided by the key strand copy/paste and mirror component. After selecting the triangles for a wisp, the geometry of a key strand can be copied or mirrored from an existing wisp to a new one as illustrated in figure 6 . 


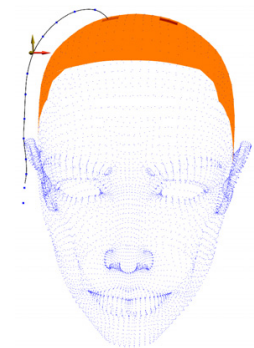

The source wisp

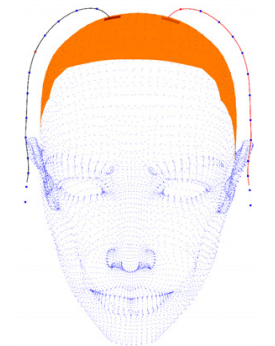

The mirrored wisp
Figure 6: The red key strand in the right image is a mirror version of the key strand in the left image.

The wisp generation component is able to generate all hair strands determined by their key strands and to distribute all hair strands over the scalp uniformly based on the hair density specified for different scalp regions.

The hair strands within a wisp tend to be similar, although some variations are required to improve realism. Choe and Ko observed that the degree of similarity can be controlled by a length distribution, radius distribution and strand variation (Choe and Ko, 2005). The length distribution determines the length variance between the key strand and a member strand within a wisp. The radius distribution controls the distance between the key strand and a member strand within a wisp. Finally the strand distribution gives the shape variation of each strand compared to the key strand.

Rather than varying the position of each control point of a member strand with respect to the key strand (Choe and Ko, 2005) we define for each control point an offset vector which linearly increases in length for each subsequent control point. The initial offset vector is randomly selected using a uniform distribution over a sphere. In order to maintain the overall shape of the strand the offset vector is defined with respect to a torsion minimising reference frame for the spline curve representing the strand (Bloomenthal, 1990).

Figure 7 illustrates this process. The key strand on the right is reproduced at the new root position. We then define a random offset vector for the first control point subject to a maximum length indicated by the circle in the figure. The offset vector's length linearly increases for subsequent control points. Applying it with respect to the key strand's reference frame generates a new curve of similar appearance. We also implemented a strand distribution but found that the hair styles using it were indistinguishable from the ones using just length and distance variations.

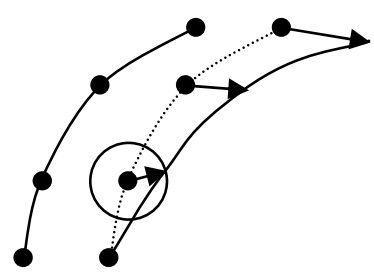

Figure 7: The original key strand (left) and the resulting member strand (right).

\section{RENDERING ALGORITHMS}

We have developed two rendering algorithms which take advantage of new GPU functionalities in order to render hair realistically in real-time. Both algorithms implement an anisotropic reflection model for the illumination of hair strands (Kajiya and Kay, 1989) and use opacity shadow maps (Kim and Neumann, 2001) for simulating self-shadowing. The algorithms differ in terms of data structures and utilised GPU features. More details are described in the implementation section and a comparison and evaluation is given in the result section.

\section{ANTIALIASING}

Because hairs are very fine structures with a width and inter-hair distance of equal or below the image pixel size, rendering hair can result in aliasing artefacts. While increasing the sampling frequency would help this is not an option when interactive performance is required and we have to employ instead antialiasing techniques which can be grouped into three approaches: prefiltering, supersampling, and postfiltering.

Prefiltering is the best way to perform antialiasing because it removes high frequencies in the view of a scene before rendering an image. This can be achieved by calculating for each rendered primitive its coverage of an image pixel. If the coverage is below $100 \%$ the values for all primitives contributing to that pixel are averaged. Analytical prefiltering removes high frequencies of the intensity function representing the image by using mathematical operators (Chan and Durand, F., 2005). Supersampling uses an increased sampling frequency. High frequency components are removed using a low pass filter (e.g. a box filter), before down-sampling the image to the required size. 
Postfiltering also uses convolution kernels, but operates directly on the final resolution image.

Our hair rendering antialiasing implementation is based on supersampling and postfiltering techniques implemented on the GPU and is described in more detail in the next section.

\section{IMPLEMENTATION}

\subsection{Rendering Algorithms}

We have implemented two rendering algorithms which both have three components: data preparation, which converts data into the required format and loads it into memory, opacity shadow maps (OSM) generation and hair rendering.

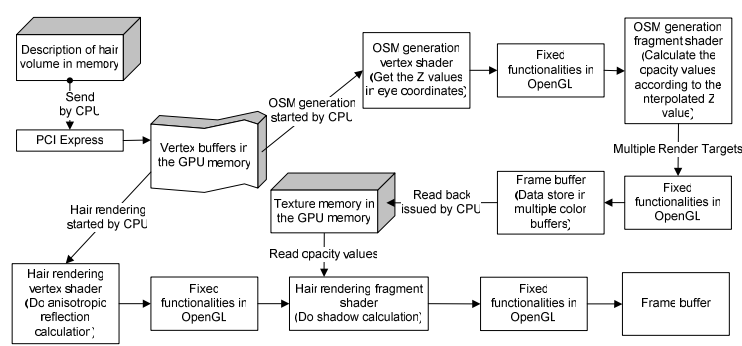

Figure 8: Overview of the first rendering algorithm.

Figure 8 illustrates our first implementation which uses vertex buffer objects to store hair data as OpenGL server side objects on the graphics card. The GPU can access the vertex buffer objects directly without transferring the data set from the CPU. Opacity shadow maps are created from the vertex buffer objects by rendering the hair geometry using the light position as view point. The fragment shader outputs the opacity value sequentially to the frame buffer through the fixed function pipeline of OpenGL by using the Multiple Render Targets technology which allows a fragment shader to write to up to four buffers within the frame buffer. Sixteen maps can be generated in one rendering pass as we store up to four maps in four channels of each buffer.

Hair strands are rendered using vertex shaders which implement an anisotropic reflection model (Kajiya and Kay, 1989) to compute the correct illumination at the polylines' vertices. The fixed OpenGL functionalities interpolate the colour values and then pass the values to the fragment shader. The shader computes the opacity value of each strand's incoming fragment by linearly interpolating the two adjacent shadow maps loaded from the GPU texture memory (Koster, Haber and Seidel, 2004). We use the following fast interpolation function which avoids branch statements for searching (Nguyen and Donnelly, 2005):

$$
\Omega=\sum_{i=0}^{n-1}\left[\Omega_{i}^{*} \max \left(0,1-\left|z-z_{i}\right| / d z\right)\right]
$$

$\Omega$ is the opacity value of a point, $\Omega_{i}$ is the corresponding opacity value on the $\mathrm{i}^{\text {th }}$ map, $\mathrm{n}$ is the total number of maps, $z_{i}$ is the distance of $\mathrm{i}^{\text {th }}$ map from the light position, $z$ is the distance of the point from the light position, and $d z$ is the distance between two adjacent maps using the assumption that all the distances between maps are equal. We have proven the validity of this formula (Anonymous, 2006).

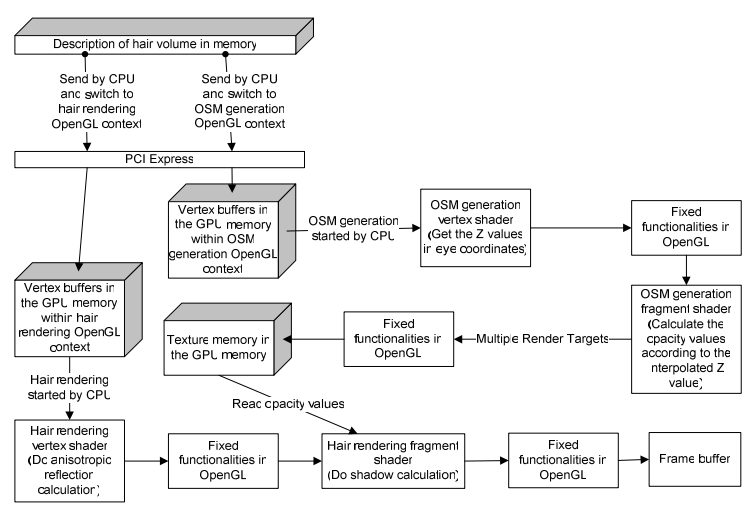

Figure 9: Overview of the second rendering algorithm.

The second rendering algorithm, outlined in figure 9, uses two OpenGL contexts with copies of the hair data in order to make use of the render-totexture technology: one copy is for the opacity shadow map generation and one for the hair rendering. The render-to-texture technology is currently the only way in GPU programming to enable a fragment shader to output data directly to the texture memory on the GPU. Hence this is the fastest way to put the data of the opacity shadow maps into the texture memory. The OSM is generated as for the first implementation, except that the fragment shader outputs the data directly into the texture memory.

The advantage of the first implementation is that it always runs in the same OpenGL context, i.e. no expensive OpenGL context switching is required. Drawbacks are the cost of copying data from the frame buffer to the texture memory and that it requires more powerful GPUs which offer multiple 
render targets. In addition the sample rate of the shadow maps is fixed to the screen resolution of the OpenGL context. The second implementation is more widely supported by graphics hardware. However, the cost for OpenGL context switching is high and the amount of memory consumed is almost 1.4 times as large as for the first implementation.

\subsection{Antialiasing}

Postfiltering is performed by copying the rendered image from the frame buffer to the texture memory in the GPU. Fast processing is achieved by using a fragment shader which averages pixel values according to the selected convolution kernel. The resulting colour of the fragment is processed by the fixed functionality OpenGL pipeline and written to the frame buffer. We restrict processing of fragments to the minimal quadrilateral covering the hair region since skin and other scene objects don't have the same fine structures as hair.

Supersampling is similar to postfiltering but differs in three points: (a) We need to adjust the OpenGL context to a larger screen size in order to accommodate the high resolution supersampled image. The highest possible resolution is depended on the capability of the graphics hardware. (b) We use the "render-to-texture" capability of modern GPUs to render to the texture memory of the GPU directly instead of copying the data back from the frame buffer to the texture memory. (c) In order to apply the convolution kernel to the incoming fragment we need to find the texture coordinates of all the neighbour pixels required by the kernel. The texture coordinates can be computed by considering the ratio between the supersampling resolution and the rendering resolution.

\section{RESULTS}

\subsection{Effectiveness}

Our hair styling toolset is capable of creating a variety of moderately complex styles. Depending on the complexity of a new hair style it can take up to several hours for a user without modelling experience to create it. Adjusting the key strands is the most time consuming step when making a specific style. Two examples of completed hair styles created by us are shown in figure 10. Our hair styling toolset can model and render real hair styles effectively as demonstrated in figure 11. Shadow effects in hair volumes are compared in figure 12.
We tested our tool with non-expert users and found that most functions such as wisp copy/paste, mirror, and preview are quite intuitive. However users found that they need to explicitly design the wisp interactions and it is a little bit difficult to define the directions of key strands. The current version of our toolkit does not perform collision detection between strands and wisps and does not use an explicit physical model and it is therefore difficult to model braided hairstyles.

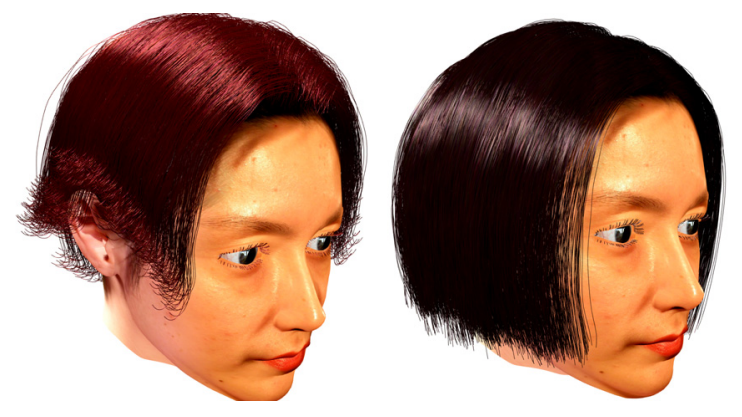

Figure 10: A curly short hair style (left) and a smooth medium length hair style (right).

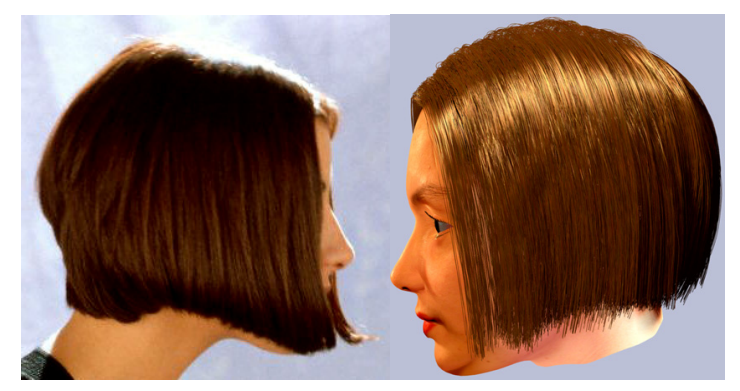

Figure 11: A real (left) and a computer generated hair style (right).

The image on the left of figure 13 demonstrates that the distribution function by Choe and Ko, which uses random offsets for control points, can lead to slightly wavy strands even if the original key strand is uniformly curved. In contrast our function produces uniformly smooth wisps. Furthermore by defining the maximum length of the initial offset vector we can produce very smooth hair where the strands are virtually parallel and very fuzzy hair where the distance between hair strands increases at the end of a wisp. 

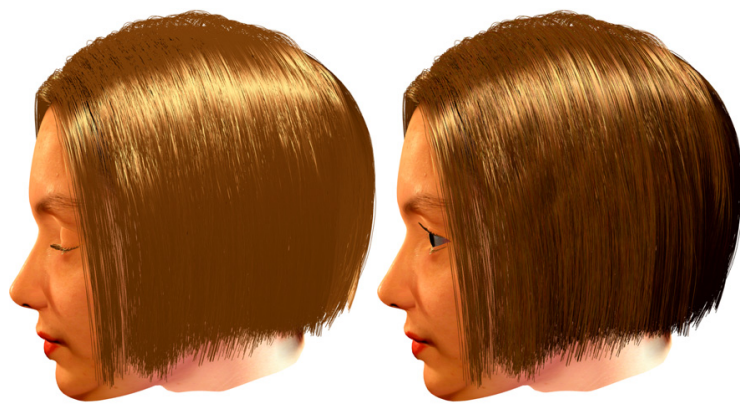

Figure 12: A hair volume without shadow (left) and with shadow (right).
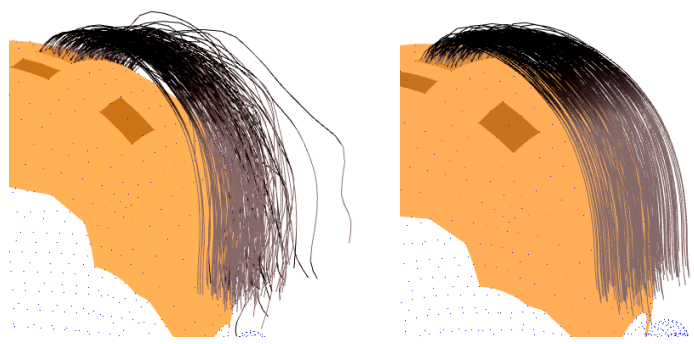

Figure 13: A large wisp generated with Choe and Ko's distribution function (left) and with our function (right). Note that the results were exaggerated by using large offset vectors in order to emphasize the differences between the methods.

\subsection{Efficiency}

We implemented our algorithms using $\mathrm{C} / \mathrm{C}++$ and OpenGL and run them on a PC with 2GB memory, a $2 \mathrm{GHz}$ Intel Pentium $\mathrm{M}$ Processor 760 and an NVIDIA GeForce Go 7800 GTX graphics card with $256 \mathrm{MB}$ memory. We found that the speed of our GPU-based anisotropic reflection algorithm is almost ten times as fast as for the equivalent $\mathrm{CPU}$ implementation (Anonymous, 2006). The complete algorithms including opacity shadow maps can render a head model with 16,215 hair strands (357,267 line segments) with 13-20 frames/second.

\subsection{Antialiasing}

We implemented GPU-based box filter and Gaussian filter kernels and found that they are able to partially solve the aliasing problem as illustrated in the images 2 and 3 of figure 14 . We found that two times hardware build-in supersampling with and without Gaussian filtering (image 5 and 6 of figure 14 , respectively) yields the best antialiasing results. Note that the images show different hair regions, but in this subsection we are only interested in the appearance of individual hairs on skin so that this does not matter.

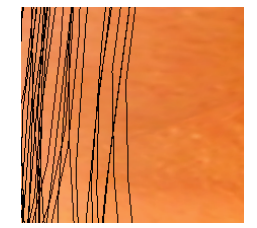

(1) Aliasing
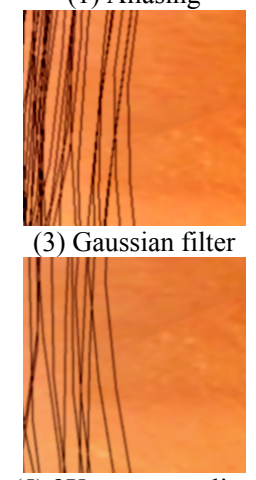

(5) $2 X$ supersampling

(Hardware build-in) and

Gaussian filter

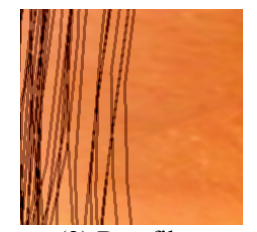

(2) Box filter

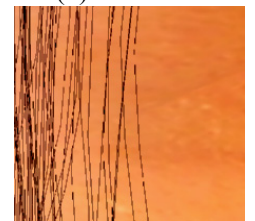

(4) $2 \mathrm{X}$ supersampling(GPU)

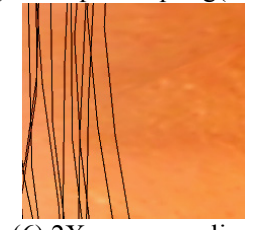

(6) $2 X$ supersampling (Hardware build-in)
Figure 14: Results obtained with different antialiasing techniques.

The performance of all antialiasing schemes is satisfactory and increases total rendering time between $17 \%$ (box filter) and $34.2 \%$ (2x hardware built-in supersampling and Gaussian filter).

\section{CONCLUSIONS}

Although the hair styling process can require a couple of hours we found that our toolset enables users to create a variety of hair styles efficiently and effectively. The toolset provides not only high-level functionality such as copy/paste and mirroring of wisps, but also low-level modifications such as changing the number and positions of a key strand's control points in order to modify the shape of a wisp. This was achieved using a novel interaction tool which uses a local-coordinate system for defining "styling directions".

We have introduced a new statistical method to generate strands from a key strand which has the advantage that it maintains consistency of style within a wisp and that it enables users to model smooth, fuzzy and fringy hair. With our density based hair distribution facility the roots of hair strands are distributed evenly over the scalp.

Rendering is performed in real-time using GPU accelerated algorithms and the whole modelling process is interactive. 


\section{FUTURE WORK}

Much work remains to be done in order to make our model suitable for a wider range of applications. Hair dynamics must be implemented in order to use our model for animations where the hair moves dynamically with the body motion or through outside forces such as wind. We believe an interesting approach for interactive environments is to create a global deformation field for the hair volume rather than modelling the motion of individual wisps. We would also like to improve the styling toolset to incorporate constrained hair styles such as ponytails and braided styles.

\section{REFERENCES}

Anjyo, K., Usami, Y., Kurihara, T., 1992. A simple method for extracting the natural beauty of hair. SIGGRAPH '92: Proceedings of the 19th annual conference on Computer graphics and interactive techniques, ACM Press, New York, NY, USA, pp. 111-120, 1992.

Anonymous, 2006, details deleted for this review.

Bloomenthal, J., 1990. Calculation of reference frames along a space curve. Graphics Gems Vol. 1, Academic Press, San Diego, CA, USA, 1990, pp. 567-571.

Catmull, E., Rom, R., 1974. A Class of Local Interpolating Splines. Computer Aided Geometric Design, R. E. Barnhill and R.F. Riesenfeld ed., Academic Press, New York, 1974, pp. 317-326.

Chan, E., Durand, F., 2005. Fast Prefiltered Lines. GPU Gems 2, Addison-Wesley, Boston, MA, USA, March 2005, pp. 345-359.

Choe, B., Ko, H., 2005. A statistical Wisp Model and Pseudophysical Approaches for Interactive Hairstyle Generation. IEEE Transactions on Visualization and Computer Graphics, vol. 11, no. 2, pp. 160-170, MarApr 2005.

Daldegan, A., Thalmann, N. M., Kurihara, T., Thalmann, D., 1993. An integrated system for modelling, animating, and rendering hair. Proceedings of Eurographics, vol. 12, pp. 211-221, 1993.

Kajiya, J.T., Kay, T.L., 1989. Rendering Fur with Three Dimensional Textures. SIGGRAPH Proceedings, vol. 23, pp. 271-280, July 1989.

Kim, T., Neumann, U., 2001. Opacity Shadow Maps. SIGGRAPH '02: Proceedings of the 12th Eurographics Workshop Rendering, June 2001, pp. 177-182.

Kim, T., Neumann, U., 2002. Interactive multiresolution hair modelling and editing. SIGGRAPH '02: Proceedings of the 29th annual conference on Computer graphics and interactive techniques, ACM Press, New York, NY, USA, pp. 620-629, July 2002.

Koster, M., Haber, J., Seidel, H., 2004. Real-Time Rendering of Human Hair using Programmable
Graphics Hardware. Proceedings of Computer Graphics International, 2004, pp. 248-256.

Lafrance, M., 2005. First Impressions and Hair Impressions. Unpublished manuscript, Department of Psychology, Yale University, New Haven, Connecticut. http://www.physique.com/sn/sn_yalestudy2.asp, visited on 15th July 2005.

Nguyen, H., Donnelly, W., 2005. Hair Animation and Rendering in the Nalu Demo. GPU Gems 2, AddisonWesley, Boston, MA, USA, March 2005, pp. 361-380.

Parke, F. I., 1974. A Parametric Model for Human Faces. Unpublished manuscript, $\mathrm{PhD}$ thesis, University of Utah, Salt Lake City, UT, UTEC-CSc-75-047, December 1974.

Perlin, K., 1989. Hypertexture, SIGGRAPH Proceedings, vol. 23, pp. 253-262, 1989.

Plante, E., Cani, M. P., Poulin, P., Perlin, K., 2001. A layered wisp model for simulating interactions inside long hair. Proceedings of Eurographics Computer Animation and Simulation 2001, pp. 139-148, Sep 2001.

Rosenblum, R., Carlson, W., Tripp III, E., 1991. Simulating the structure and dynamics of human hair: Modelling, rendering and animation. The Journal of Visualization and Computer Animation, vol. 2, no. 4, pp. 141-148, October-December 1991.

Stam, J., 1995. Multi-Scale Stochastic Modelling of Complex Natural Phenomena. PhD Thesis, Dept. of Computer Science, University of Toronto.

Yang, X. D., Xu, Z., Yang, J., Wang, T., 2000. The Cluster Hair Mode. Graphical Models, vol. 62, pp. 85103, 2000. 